\title{
Noise in Piezoresistive and Capacitive Sensors
}

\author{
Richard R. Spencer, Bruce M. Fleischer, Phillip W. Barth, and James B. Angell
}

\begin{abstract}
An analysis has been performed of the minimum resolvable input $(M R I)$ for piezoresistive and capacitive pressure sensors as determined by fundamental noise mechanisms. Even though capacitive sensors have raw sensitivities that are an order of magnitude or more higher than those of piezoresistive sensors, the MRI of piezoresistive sensors (ignoring temperature coefficients and offsets) can be much lower than that of comparable capacitive sensors. Piezoresistive sensors may thus be more promising than capacitive sensors for applications requiring a large dynamic range $(D R)$ and/or small $M R I$.
\end{abstract}

\section{Glossary of Symbols}

\begin{tabular}{|c|c|}
\hline$B_{m}$ & Measurement bandwidth (Hertz) \\
\hline$C$. & Capacitance of a capacitive pressure sensor (Farads) \\
\hline$d$ & $\begin{array}{l}\text { Distance between plates of capacitor with } P=0 \\
(\mu m)\end{array}$ \\
\hline$D R$ & Dynamic range $\left(P_{\max } / M R I\right)$ \\
\hline$E$ & Young's modulus (mmHg) \\
\hline$h$ & Diaphragm thickness $(\mu m)$ \\
\hline$k$ & Boltzmann's constant (Joules/K) \\
\hline$k T$ & Thermal energy of one electron (Joules) \\
\hline$L$ & Length of one side of a square diaphragm $(\mu m)$ \\
\hline$M R I$ & Minimum Resolvable Input (mmHg) \\
\hline$P$ & Pressure on diaphragm (mmHg) \\
\hline$P_{\max }$ & Maximum allowable input pressure ( $\mathrm{mmHg}$ ) \\
\hline$q$ & charge on an electron (Coulombs) \\
\hline$R$ & Resistance $(\Omega)$ \\
\hline$r$ & Radius of a circular diaphragm $(\mu m)$ \\
\hline$S_{\text {cap }}$ & $\begin{array}{l}\text { Capacitive sensitivity, defined as change in ca- } \\
\text { pacitance divided by reference-pressure capacitance } \\
\text { (ppm/mmHg) }\end{array}$ \\
\hline$S_{\text {piezo }}$ & $\begin{array}{l}\text { Piezoresitive sensitivity, defined as change in re- } \\
\text { sistance divided by reference-pressure resistance } \\
(\mathrm{ppm} / \mathrm{mmHg})\end{array}$ \\
\hline$t$ & time \\
\hline$T$ & Absolute temperature $(\mathrm{K})$ \\
\hline$V_{b}$ & Bias voltage applied to sensor (V) \\
\hline$\epsilon$ & Permittivity of dielectric used for $C_{s}$ and $C_{r}(F / \mu m)$ \\
\hline $\begin{array}{l}\nu \\
\sigma_{\text {fract }}\end{array}$ & $\begin{array}{l}\text { Poisson's ratio } \\
\text { Fracture stress of silicon }(\mathrm{mmHg})\end{array}$ \\
\hline$\sigma_{\max }$ & Maximum stress in diaphragm (mmHg) \\
\hline
\end{tabular}

This work was supported by Ford Corporation and the National Institutes of Health. Bruce Fleischer is supported by an AT\&T Bell Laboratories PhD scholarship.

R. Spencer and B. Fleischer are graduate students at Stanford. Their mailing address is AEL 124, Stanford University, Stanford, CA 94305.

J. Angell is the Associate Chairman of the department of Electrical Engineering at Stanford. His mailing address is McCullough 162, Stanford University, Stanford, CA 94305.

P. Barth was with Stanford, he is now the Program Director, Special Sensor Development, at NovaSensor, 2975 Bowers Ave., Santa Clara, CA 95050.

\section{Introduction}

The purpose of this investigation is to determine the minimum detectable signal for a pressure sensor assuming that all systematic errors (e.g., temperature coefficient and offset) have been eliminated. Capacitive and piezoresistive sensors are classified based on the method used to measure $\Delta \mathrm{C}$ or $\Delta \mathrm{R}$. Noise in the detection and bias circuitry has been separated from noise in the sensor element proper where possible. In some configurations, however, circuit noise is an inherent part of the sensing method and so is included in the analysis.

Several comparisons of piezoresistive and capacitive sensors have been published (e.g., $[1,2,3])$. Only the work by Chau and Wise [3] deals specifically with noise mechanisms, and that work did not consider the effects of different measurement techniques. Chau and Wise did show that Brownian noise is insignificant in all practical cases and it will not be considered here.

\section{Capacitive Sensors}

Chau and Wise imply that $\mathrm{kT} / \mathrm{C}$ noise is dominant in all capacitive sensors. However, for many capacitive sensors $\mathrm{kT} / \mathrm{C}$ noise is confined to a narrow bandwidth and does not affect the achievable resolution. Depending on the measurement technique used, the limit is determined by either shot noise integrated by the capacitor, $\mathrm{kT} / \mathrm{C}$ noise, or noise in the switching operation (if a ratiometric scheme is used).

Detection circuitry for capacitive sensors often uses a current-controlled oscillator $[4,1]$. Since the currents used to ramp the oscillator must be switched, the dominant noise mechanism will be determined by the type of switches used. If the switches are MOS devices then the analysis is the same as for the $\mathrm{RC}$ oscillator circuit described below. If the switches are bipolar devices then their shot noise determines the fundamental limit to the performance.

With bipolar switches the only $\mathrm{kT} / \mathrm{C}$ noise present is due to the resistance of the dielectric and has a bandwidth on the order of $10^{-3} \mathrm{~Hz}$. The lower limit of the measurement bandwidth is much greater than the upper limit of the noise bandwidth and $\mathrm{kT} / \mathrm{C}$ noise is not detectable. The minimum resolvable input for this shot noise limited case is

$$
M R I=\frac{2}{S_{c a p}} \sqrt{\frac{q}{C_{s} V_{b}}} .
$$

The plate-touching pressure is

$$
P_{\max }=\frac{5.33 d E h^{3}}{r^{4}\left(1-\nu^{2}\right)}
$$

and the dynamic range is

$$
D R=0.295 r \sqrt{\frac{\epsilon V_{b}}{d q}} .
$$

Only two of these three quantities are independent (the dynamic range is defined as the maximum input pressure divided by the $M R I) . P_{\max }$ is derived from the circular-diaphragm sensitivity equation in [3] and the equation for the deflection at the center of the diaphragm in [5]. 
Equations have also been derived for capacitive sensors where $\mathrm{kT} / \mathrm{C}$ noise is the dominant mechanism (e.g., $\mathrm{RC}$ relaxation oscillators). The maximum input pressure is the same as above and the other equations are

$$
M R I=\frac{5.44}{S_{c a p} V_{b}} \sqrt{\frac{k T}{C_{s}}}
$$

and

$$
D R=.167 V_{b} \sqrt{\frac{C_{s}}{k T}} .
$$

These equations were derived by assuming that $t=R C_{s}$ is the parameter measured. The jitter in $t$ was found using a method similar to Abidi's [6].

If a ratiometric measurement scheme is used then the $M R I$ is determined by the circuit implementation. In a simple MOSswitch charge-sharing approach the $\mathrm{kT} / \mathrm{C}$ noise resulting from the switch resistance is dominant. If more complicated switched capacitor schemes are used then the MRI is determined by op-amp noise, random errors in charge cancellation, and other sources determined by the particular design. Although promising, this technique is more complicated than the others discussed and no sensors using it have been described in the literature.

\section{Piezoresistive Sensors}

For piezoresistive sensors two general measurement techniques are possible: a ratiometric scheme (including the classic bridge configuration), or an $\mathrm{RC}$ oscillator. In both cases the dominant noise mechanism is thermal noise in the piezoresistor. $P_{\max }$, defined to be the rupture pressure, is found using equations for $S_{\text {piezo }}, \sigma_{\text {peak }}$, and $\sigma_{\text {fract }}[1]$.

$$
P_{\max } \approx 8.96 \times 10^{6}\left(\frac{h}{L}\right)^{2}
$$

For piezoresistive RC sensors the $M R I$ is the same as noted above for capacitive RC sensors except that the appropriate sensitivity must be used:

$$
M R I=\frac{5.44}{S_{\text {piezo }} V_{b}} \sqrt{\frac{k T}{C}},
$$

and

$$
D R \approx .03 V_{b} \sqrt{\frac{C}{k T}} .
$$

For the ratiometric piezoresistive sensor (assuming a standard bridge configuration)

$$
M R I=\frac{2 \sqrt{4 k T R B_{m}^{-}}}{S_{\text {piezo }} V_{b}},
$$

and

$$
D R \approx \frac{.022 V_{b}}{\sqrt{4 k T R B_{m}}}
$$

\section{Summary}

Using these equations it is possible to compare the performance limits of capacitive and piezoresistive sensors. These equations also show how the $M R I, D R$, and $P_{\max }$ scale as long as the assumptions used in deriving them remain valid. The table below compares the calculated MRI for four representative sensors (adapted from [3]).

\begin{tabular}{|l|c|c|c|c|}
\hline \multicolumn{2}{|c|}{ Minimum Resolvable Input $^{1}$ (mmHg) } \\
\cline { 2 - 5 } & \multicolumn{2}{|c|}{ Ultraminiature } & \multicolumn{2}{c|}{ Ultrasensitive } \\
\cline { 2 - 5 } & Piezo (C1) & Cap (A1) & Piezo (D1) & Cap (B1) \\
\hline Ratiometric & $1.6 \times 10^{-4}$ & see note 2 & $7.1 \times 10^{-6}$ & see note 2 \\
\hline RC Oscillator & $5.9 \times 10^{-4}$ & $1.7 \times 10^{-2}$ & $2.8 \times 10^{-4}$ & $1.0 \times 10^{-2}$ \\
\hline Current Controlled Oscillator $^{3}$ & N/A & $6.7 \times 10-2$ & N/A & $4.1 \times 10^{-2}$ \\
\hline
\end{tabular}

Notes:

1. Unless stated otherwise, a $3 \mathrm{~V}$ supply and a $100 \mathrm{~Hz} B_{m}$ have been used.

2. Depends on exact circuit configuration, see text.

3. These numbers do not use a $100 \mathrm{~Hz} B_{m}$ and can be improved slightly. How much improvement is possible depends entirely on circuit design and device performance, but the $M R I$ will always be larger than for the piezoresistive case.

\section{Acknowledgements}

We thank Lyn Bowman for his participation in the early discussions of this work.

\section{References}

[1] Craig S. Sander. A Bipolar-Compatible Monolithic Capacitive Pressure Sensor. PhD thesis, Stanford University, December 1980.

[2] Y. S. Lee. A Silicon Capacitative Pressure Transducer: Performance Characteristics and Limitations. $\mathrm{PhD}$ thesis, University of Michigan, December 1981.
[3] H. L. Chau and K. D. Wise. Scaling limits in batchfabricated silicon pressure sensors. In Transducers'85, pages 174-177, IEEE, 1985.

[4] Michael John Sebastian Smith. An Integrated Circuit for a Biomedical Capacitive Pressure Transducer. $\mathrm{PhD}$ thesis, Stanford University, June 1985.

[5] Wen H. Ko and Min-Hang Bao, and Yeun-Ding Hong. A high-sensitivity integrated-circuit capacitive pressure transducer. IEEE Trans. on Electron Devices, ED-29(1):48-56, Jan 1982.

[6] Asad A. Abidi and Robert G. Meyer. Noise in relaxation oscillators. IEEE Journal of Solid State Circuits, SC-18(6): 794-802, December 1983. 